\title{
Urban Citizen Walkers
}

Methodologische Reflexionen zum kollaborativen Gehen in der Stadt

\author{
Katharina Rohde
}

Kathrin Wildner

\section{Start walking}

Alle Welt geht. In der Literatur (Cole 2012; Cadogan 2016), der Kunst (Kunstforum International Nr. 266, 2020) und in Ausstellungen („Des marches, démarches“, Marseille 2020), aber auch in der wissenschaftlichen Praxis (Ingold 2008; Solnit 2000; Brown/Shortell 2016; Lisiak et al. 2019; Nguyen 2016). Die Auseinandersetzung mit dem Gehen ist (immer wieder) aktuell. Meist geht es bei diesen Gehversuchen um eine Verbindung des Körpers mit dem umgebenden Raum (z. B. Debord 1990; Pétonnet 2017) oder um die Verschiebung der räumlichen und zeitlichen Maßstabsebene (vgl. Augoyard 2007, 2017), die der Geschwindigkeit des globalen Zeitalters etwas entgegenzusetzen vermag (Rolshoven 2017). Seltener geht es in den Beiträgen um eine systematische Betrachtung von Gehen als Forschungsmethode oder gar um das Gehen als eine transformative Praxis der Stadtproduktion.

Als Stadtforscherinnen und urban practitioners[1] möchten wir in diesem kollaborativen Text die Gelegenheit nutzen, das Gehen als eine methodische Praxis der kritischen Stadtforschung zu ergründen. Wir verstehen das Gehen als ein Werkzeug, die Komplexität von Stadt vor dem Hintergrund unterschiedlicher Erfahrungen zu begreifen. Vor allem aber verstehen wir das Gehen als ein mögliches Mittel der Raumaneignung beziehungsweise der aktiven und selbstermächtigten lokalen Verortung und damit der (temporären) Selbst-Einschreibung in den urbanen Raum.

Die Methode des kollaborativen Gehens als Möglichkeit der Teilhabe an Stadtgesellschaft ist nicht neu und wird auch - in Berlin und anderswo - bereits angewandt. So veranstaltete das Deutsche Architekturzentrum (DAZ) in Berlin 2015 und 2016 Stadtspaziergänge[2] zusammen mit Architekt*innen, Planer*innen, Künstler*innen und geflüchteten Menschen. Sie zielten darauf ab, den neu Angekommenen eine Orientierung in der Stadt, einen Zugang zu städtischen Institutionen zu ermöglichen und darüber hinaus persönliche Kontakte und sogar berufliche Anknüpfungspunkte zu generieren. Die fortlaufenden Refugee Voices Tours[3] werden hingegen von geflüchteten Menschen organisiert und vermitteln einen Einblick in persönliche 
Geschichten und Erlebnisse vor, während und nach der Flucht (vgl. Rohde/ d'Auria 2018).

Was wir mit unseren Urban Citizen Walks hinzufügen möchten, ist der Versuch, die Stadt post-migrantisch und im Sinne einer pluralistischen Gesellschaft zu gestalten. Post-migrantisch heißt, dass wir uns mit politischen und gesellschaftlichen Aushandlungsprozessen, die nach der Migration erfolgen und hinter der Migrationsfrage versteckt bleiben, auseinandersetzen (Foroutan 2019). Dabei scheint uns bezüglich dieser politischen und gesellschaftlichen Aushandlungsprozesse auf lokaler, urbaner Ebene die Frage nach urban citizenship zentral. Es geht uns nicht darum, Orientierungshilfe zu leisten, sondern gemeinsam mit unterschiedlichen Stadtbewohner*innen Urban Citizen Walks zu initiieren und uns die Stadt anzueignen, im Idealfall Orte (neu) zu besetzen.

Als kollaboratives Experiment organisieren wir daher im Sommer 2019 zusammen mit Menschen unterschiedlicher Herkunft, die seit kurzem oder schon lange in Berlin leben, eine erste Stadterkundung. Darin betrachten wir als Urban Citizen Walkers die Stadt aus der Perspektive des Ankommens mit dem Blick des Sich-Zurechtfindens in alltäglichen Momenten der Aneignung.

In diesem Beitrag gehen wir der Frage nach, wie das Gehen als Methode der kritischen Stadtforschung hin zu einer aktiven Produktion einer urban citizenship weiterentwickelt werden kann. Dazu werden wir zunächst einige theoretische und methodologische Aspekte des Gehens vorstellen. Anschließend beschreiben wir das von uns entwickelte Format der kollaborativen Stadterkundung, wobei wir uns an dieser Stelle auf die methodologische Reflexion konzentrieren. Abschließend diskutieren wir die Potenziale dieser Methode als Praxis zur Teilhabe an der alltäglichen Produktion von Stadt ganz im Lefebvre'schen Sinne als materieller, sozialer, diskursiver Raum und damit als eine mögliche Voraussetzung für urban citizenship.

\section{Gehen als ... - Theoretische Konzepte}

Das Gehen wird in der qualitativen Stadtforschung inzwischen zu den zentralen Momenten der explorativen Analyse gezählt. Ohne den state of the art in Bezug auf das Gehen wiederholen zu wollen,[4] möchten wir doch einige für unser Vorhaben zentrale Autor*innen und deren Texte erwähnen. Hierbei legen wir unseren Fokus auf interdisziplinäre Ansätze mit dem Ziel, die Potenziale des Gehens über Merkmale der ästhetischen Form und der Alltagspraxis hinaus als Forschungswerkzeug und Instrument der Wissensproduktion, vor allem aber als Mittel der Raumaneignung, zu postulieren. Im Sinne des Experimentes folgen diese Ausführungen den Rhythmen des Umherschweifens, des Abdriftens, des Flanierens.

\section{Gehen als ästhetische Praxis}

Ein Ursprung des Gehens als ästhetischer Praxis in der Stadt wird mit der literarischen Figur des Flaneurs gesetzt. Walter Benjamin widmet ein ganzes Kapitel seines Passagen-Werkes dem Flaneur und sammelt Versatzstücke und Fragmente, etwa die kuriose Bemerkung, dass es im 19. Jahrhundert in Mode war, beim Flanieren eine Schildkröte mit sich zu führen 
(Benjamin 1998). In diesem Bild zeigt sich eine fast arrogante Selbstinszenierung - das Flanieren als Luxus der Langsamkeit wirft die Frage auf, wer sich überhaupt das passionierte Gehen in der Stadt leisten konnte. Dementsprechend ist es auch nicht verwunderlich, dass die romantisierenden (und bürgerlichen) Beschreibungen der Flaneure in den modernen Großstädten (Hessel 2011 [1929]; Benjamin 2015 [1935]; Krakauer 1964) heute vor allem aus feministischer und postkolonialer Perspektive kritisiert werden (u. a. Dündar et al. 2019; Cadogan 2016). Kritisch hinterfragt wird, wer überhaupt über die Möglichkeit (und den Status) verfügt, zu gehen: Frauen? Arbeiter*innen? People of Color? Denn gerade im öffentlichen Raum sind Fragen der Sichtbarkeit, Genderrollen und Machtverhältnisse präsent; hier werden Zuschreibungen und Zugänglichkeit verhandelt, die der umherschweifende Flaneur - meist ein weißer Mann - selten reflektiert.

Andererseits bedienen sich Autor*innen wie Walter Benjamin der Figur des Flaneurs gerade, um die bürgerliche, städtische Konsumwelt kritisch zu betrachten. Ganz ähnlich die Situationisten (eine Gruppe überwiegend männlicher Künstler*innen um Guy Debord), die mit dem dérive in den 1950er und 6oer Jahren eine radikale Kritik an der zunehmend sich kapitalisierenden und individualisierenden Gesellschaft formulierten und diese im städtischen Raum performativ praktizierten (vgl. Debord 1990; Sadler 1999; Grube/Thiele 2020). Sie erfanden die Methode der Psychogeographie, die das Umherschweifen in der Stadt beschreibt und mit deren Hilfe sie die Einflüsse gebauter Umwelt auf die subjektive Wahrnehmung und das soziale Verhalten untersuchten. Unter Einbezug früherer Schriften Lefebvres zu Routinen des Alltagslebens hinterfragten die Situationisten den gebauten wie gesellschaftlichen Raum. Das Gehen der Situationisten lässt sich als eine performative Praxis einer radikalen Kritik der kapitalistischen Gesellschaft beschreiben. Heute spricht man von einem künstlerischen Genre und den walking artists oder artistes marcheurs, also Künstler*innen, die das Gehen als Grundlage ihrer ästhetischen Praxis verstehen (vgl. Fischer 2011).[5]

\section{Gehen als Methode der Stadtforschung}

Aber nicht nur in der Kunst, auch in der gegenwärtigen Stadtforschung ist das Gehen eine zentrale Kategorie qualitativer Untersuchungen, eine empirische Wahrnehmungstechnik und ein ethnographisches Forschungsinstrument. In der deutschsprachigen Stadtforschung[6] ist Michel de Certeau's Text Gehen in der Stadt (1980) eine zentrale Referenz. De Certeau vergleicht das Gehen mit der Rhetorik der gesprochenen Sprache. Durch die (alltägliche) Bewegung des Gehens in der Stadt folgt der*die Fußgänger*in den urbanen Texturen, interpretiert sie und produziert damit den als relational verstandenen urbanen Raum.

Der eigene Körper wird bei de Certeau somit zu einem Instrument der Erkundung und ermöglicht eine sensitive Erfahrung im Hier und Jetzt. Nestor Garcia Canclini setzt in diesem Sinn in den 1990er Jahren bei Untersuchungen zu Mobilität in Mexiko-Stadt die Figur des Flaneurs als methodologischen Ansatz ein (Garcia Canclini 1995). Ziel des interdisziplinären Forschungsprojektes zu alltäglichen Reisen in der Stadt sind die zunächst offene und assoziative Wahrnehmung der Umgebung, die explorative, 
noch unstrukturierte Sammlung von urbanen Momenten, Treffpunkten, Ereignissen und Attraktionen und eine konzentrierte (Selbst)Reflexion des Alltagswissens der jeweiligen Personen, Flaneur*innen und Reisenden.

Einen weiteren Ansatz, das Gehen als Forschungsinstrument einzusetzen, hat Margarethe Kusenbach (2003) entwickelt. Sie führt die Methode der go-alongs ein, eine Kombination aus teilnehmender Beobachtung und Interview. Die Forscherin begleitet die Bewohner*innen auf ihren Wegen durch die Stadt. Die Potenziale sieht Kusenbach in der (gemeinsamen) Reflexion der Wahrnehmung der alltäglichen Umgebung, in der verstärkten Aufmerksamkeit auf räumliche Praktiken und soziale Interaktionen, die im Moment des go-alongs sichtbar und im Kontext der sozialen Landschaft erlebbar werden. Mittels des Gespräch werden über die konkrete Situation hinaus biographische Erfahrungen eingeflochten, die die Orte mit Lebensgeschichten verbinden. Ganz ähnlich setzt Monika Streule (2018) die Methode der ,Interviews in Bewegung zur Erkundung großräumiger Teile von Mexiko-Stadt ein, indem sie die walks mit Bewohner*innen und Expert*innen anhand von Interviewleitfäden strukturiert.

Für Colin McFarlane (2011) ist das Gehen einer der zentralen Ansätze, um etwas über den urbanen Raum, aber auch über uns selber zu lernen. Durch das Gehen werden nicht nur räumlich-urbanistische Zusammenhänge erfahrbar, sondern das Gehen sei eine temporäre Praxis, die als Teil eines umfassenderen Sets von alltäglichen Rhythmen urbane Orte charakterisierten (ebd.: 50).

Johanna Rolshoven führt in ihrem Text Gehen in der Stadt (2017) die unterschiedlichen Überlegungen zum Gehen zusammen. Sie reflektiert die Methode eng an dem Entstehen von Stadt. Neben einer konkreten Anleitung zum Gehen[7] systematisiert sie die Potenziale der Methode. Sie markiert einen spezifischen Umgang mit Zeit: In einem allgemeinen Zustand der Hypermobilität wirken die Bewegung und der Körper im Raum wie Slow Motion, ein Gegenentwurf zu Effizienz, Optimierung und Geschwindigkeit gesellschaftlicher Bedingungen. Im Weiteren beschreibt sie das Gehen als eine Form der Aneignung von Raum: Es findet im Hier und Jetzt statt, in situ. Durch die evidente Präsenz, die wahrnehmbare Anwesenheit, entsteht ein spezifisches Verhältnis zwischen Subjekt und umgebendem Raum. Und schließlich ist das Gehen Ausdruck einer selbstbestimmten Handlung. Dabei wird Handlung im Sinne Hannah Arendts (1992) verstanden als ein Prozess der Kommunikation, einer politischen Interaktion, die im öffentlichen Raum stattfindet: das Gehen im Raum als politische Haltung.

\section{Gehen als aktivistische Praxis}

Die Stadt ist ein Erfahrungsraum, dessen Potenziale und Merkmale erst durch den Gebrauch wahrnehmbar werden. Sich gehend im Raum zu bewegen, bedeutet also immer auch sich den Raum anzueignen wie auch ihn überhaupt als (sozialen) Raum herzustellen. Das Gehen schafft einen politischen Raum mit einem Potenzial der Reflexion und des Widerspruchs (Winkler 2017: 11). Agata Lisiak, Reece Cox, Flavia Tienes und Sophia Zbinovsky Braddel untersuchen dementsprechend das Gehen als kollektives Experiment aktivistischer Interventionen und subversiver Aktionen im öffentlichen Raum. 
Sie zeigen, wie das Gehen selbst zu einem Werkzeug der Positionierung und Sichtbarwerdung wird und im Gehen sogar neue kollektive und soziale Praktiken entstehen können (Lisiak et al. 2019). Anders als der meist individualisierte Flaneur wird dabei mit dem kollaborativen Gehen eine Präsenz im öffentlichen Raum evident. Das Gehen in der Gruppe unterstützt die Sichtbarkeit im urbanen Raum. Wie bei einer Demonstration - ein massives gemeinsames Gehen - aber auch darüber hinaus, bedient sich das engaged walking alltäglicher Situationen und Orte. Hierbei geht es nicht um die politische Positionierung durch eine lautstarke Masse, sondern um die unmittelbare Nutzung und kollektive Aneignung von alltäglichen Räumen als Widerstandspraxis (ebd.).

Mit dem Urban Citizen Walk testen wir, inwieweit es möglich ist, durch das kollektive Gehen Orte in der Stadt gemeinsam zu erfahren, zu lesen und (neu) zu besetzen. Wissen wird über die Geschichte(n) der Stadt, über Nutzer*innengruppen und Nutzungen und über Planung und Aneignung gemeinsam generiert. In Anknüpfung an die dargestellten Theorien des Gehens als stadtethnologische Forschungspraxis sowie als Form der politischen Positionierung fragen wir uns, ob und wie kollektive Stadterfahrungen und Selbstermächtigungsprozesse zur Teilhabe an Stadt generiert werden können und damit auch eine Zugehörigkeit zu einer städtischen Gesellschaft neu definiert werden kann.

\section{Experiment am Oranienplatz, Berlin}

„INSPIRATIONS to walk: you can walk in a circle around the square, cross it, zig-zag it, leave it for a while, find a place to hang out, walk with your eyes closed or backwards (only in pairs!), lay down for a moment, sit on a bench, jump, change your tempo, change the perspective, engage with users of the square, hold hands [...].“ (Aus der Anleitung zum Urban Citizen Walk, siehe Abb. 1)

Als Datenbasis für diesen Artikel erfinden wir das Format des Urban Citizen Walk und organisieren einen Versuch an einem Nachmittag Mitte August 2019. Die Wahl für unsere Erkundung fällt auf den Oranienplatz (O-Platz) in Berlin-Kreuzberg. Der O-Platz ist ein ,Paradebeispiel' für die Berliner Stadtentwicklung nach den Plänen von Peter Joseph Lenné Mitte des 19. Jahrhunderts. Heute ist er Symbol für Aneignungsprozesse, Proteste und Urban-Citizenship-Kämpfe, nicht zuletzt aufgrund seiner Besetzung durch geflüchtete Menschen und deren Unterstützer*innen zwischen 2012 und 2014. [8] Zu unserem Experiment haben wir zehn Menschen unterschiedlicher Herkunft eingeladen, von denen einige erst seit kurzem, andere schon länger in Berlin leben. Ahmed, Omar und Tarek kommen aus Kairo. Tarek wohnte erst in Prag und pendelte drei Jahre zwischen hier und dort. Kate ist gebürtige Engländerin, die lange in Athen lebte und jetzt hin- und herreist. Agata ist aus Polen und lebt seit vielen Jahren in Berlin. Ayşe kommt aus Istanbul, ist seit einer Weile in Marburg ansässig und kommt regelmäßig nach Berlin. Layla ist Syrerin, lebt in Berlin-Köpenick, arbeitet in Cottbus und schreibt ihre Doktorarbeit in Belgien. Hişar ist vor ein paar Jahren aus Bremen zum Studieren nach Berlin gezogen. Martin ist Rheinländer, lebt 
seit 30 Jahren in Berlin, zehn davon in Kreuzberg, und fährt jede Woche zum Arbeiten nach Braunschweig. Randa ist Syrerin aus Damaskus und zum Zeitpunkt des gemeinsamen Spaziergangs erst seit fünf Tagen in Berlin. Davor lebte sie in Kairo. Wir Autorinnen dieses Textes sind ebenso Zugezogene: Kathrin aus Westfalen, lebt nach 20 Jahren in Hamburg und Mexiko-Stadt nun seit zehn Jahren in Berlin und pendelt regelmäßig zurück nach Hamburg. Katharina, aus Schleswig-Holstein, ist seit 1997 in Berlin, allerdings unterbrochen durch längere Auslandsaufenthalte in Caracas, Johannesburg und Lagos. Aktuell lebt sie zwischen Berlin und Brüssel. Uns alle verbindet die (leidenschaftliche) Auseinandersetzung mit Städten: als Architekt*innen, Stadtforscher*innen, Kulturwissenschaftler*innen, Ethnolog*innen, Anthropolog*innen und oral historians.

Wir treffen uns mit den eingeladenen Urban Citizen Walkers am Kottbusser Tor in Berlin-Kreuzberg und gehen anschließend gemeinsam zum Oranienplatz. Die Teilnehmer*innen waren im Vorfeld über die Idee des experimentellen Spaziergangs informiert, wussten allerdings nicht, wohin wir gehen würden. Dieser, Überraschungseffekt‘ war uns wichtig, um keine Vorab-Recherche zu stimulieren, keiner Voreingenommenheit, keinen vorgefertigten Bildern und Assoziationen Raum im Kopf zu geben.

Eine von uns, den Autorinnen des Textes, im Vorfeld erarbeitete Choreografie zum Erkunden des Ortes hilft, in das Experiment einzusteigen. Um anzukommen, gehen wir erst einmal als Gruppe um den O-Platz herum. Dieses gemeinsame Gehen erzeugt eine Wirkung nach außen; wir treten als Gruppe auf, sind damit für andere als solche sichtbar und bemerken, dass wir fragend beäugt werden. Umgekehrt ist es natürlich ebenso unser Anliegen zu beobachten, und das Gesehene, Gefühlte und Gehörte (kritisch) zu reflektieren.

Nach einer gemeinsamen ersten Runde bitten wir unsere geladenen Walker, jetzt einzeln und für sich allein zu gehen. Um die Wahrnehmung anzuregen, haben wir eine ,Anleitung' formuliert, die befolgt werden kann,

Abb. 1 Mapping und Notizen eines Teilnehmers des Urban Citizen Walk auf dem von den Autorinnen ausgegeben Leitfaden, Berlin 2019
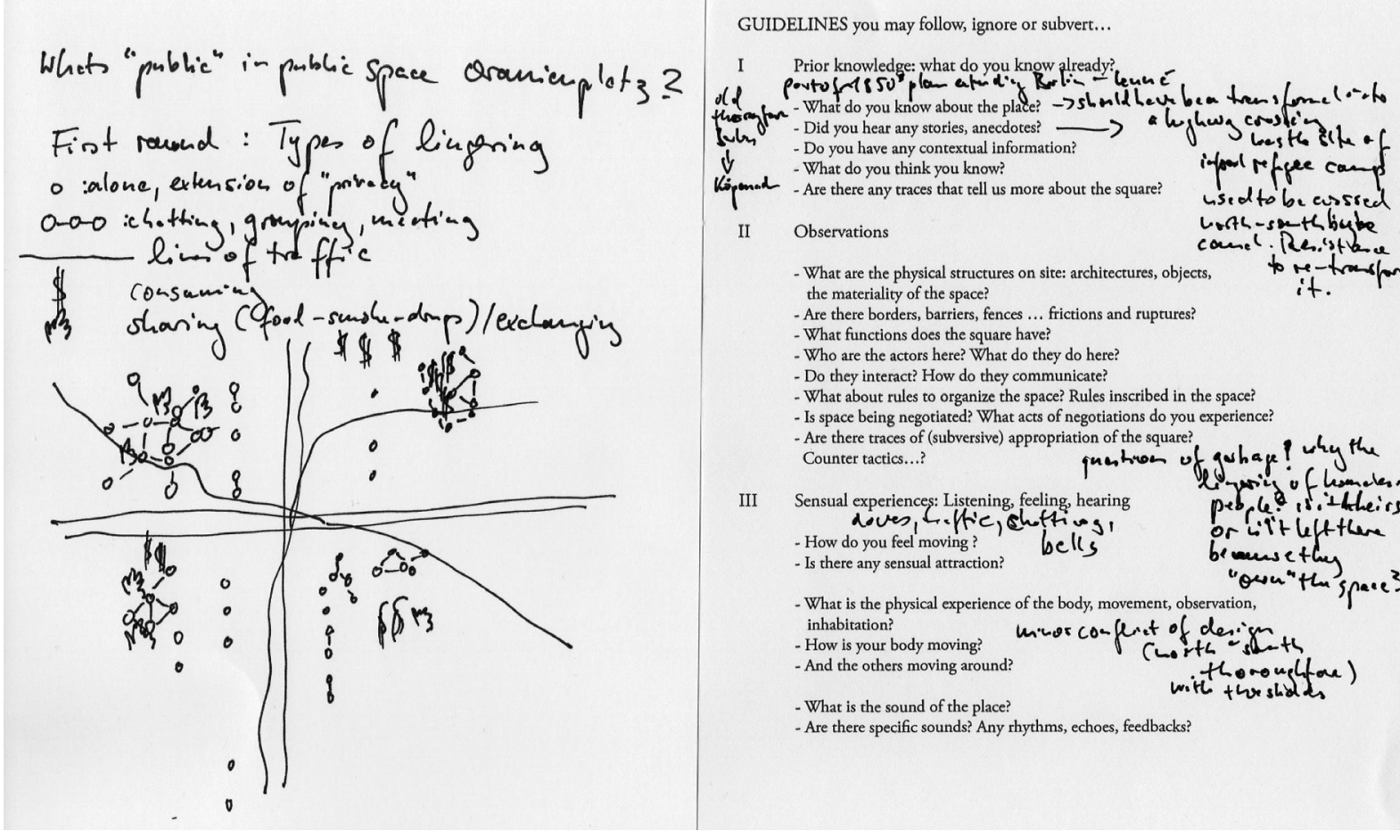

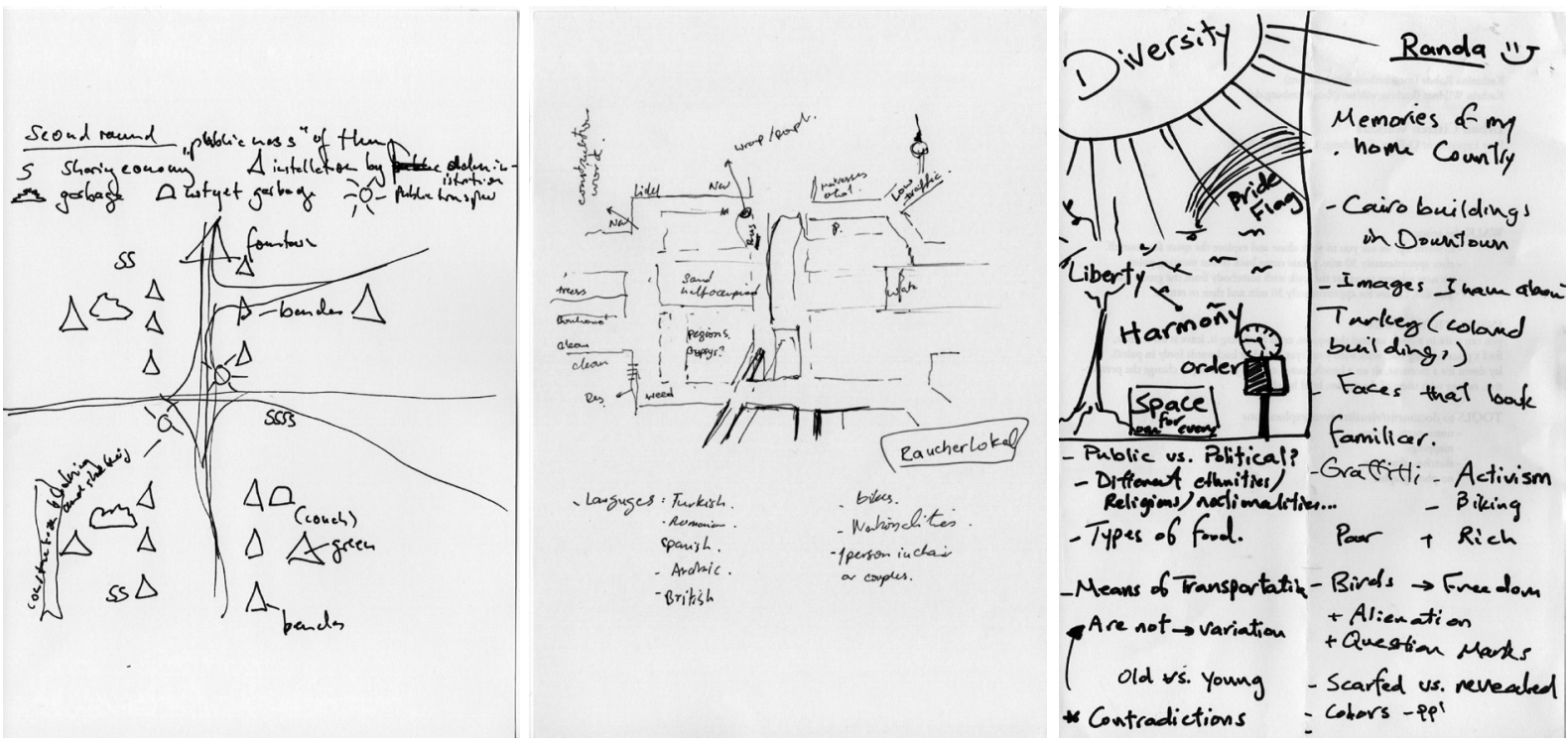

Abb. 2 bis

Abb. 4 Mappings des Oranienplatz von Teilnehmer*innen des Urban Citizen Walk, Berlin 2019 aber nicht muss, die also angeeignet, das heißt transformiert oder auch ganz ignoriert werden kann. Die einzige Bedingung ist, keine Fotos zu machen, weil wir mittels Fotografieren den Blick verändern und eher dem Bild nachgehen, als den Ort mit allen Sinnen wahrzunehmen. Unsere Anregungen zum Spazieren weisen auf ein Vorwissen zum Ort, auf das Beobachten von physischen Strukturen, Materialität, Grenzen, Funktionen, Akteur*innen, Regeln, Verhandlungen sowie auf sensorische Wahrnehmungen (Hören, Sehen, Fühlen).

Die geplanten 30 Minuten des Allein-Spazierens vergehen erstaunlich schnell, was uns verdeutlicht, wie viel es an einem (nicht allen unbekannten) Ort zu entdecken gibt. Wir treffen uns als Gruppe wieder und machen uns zu zweit erneut auf den Weg. In dieser dritten Runde werden die unterschiedlichen Perspektiven auf den Oranienplatz gemeinsam erfahren und ausgetauscht. Die Paare finden sich selbst zusammen, dürfen sich einander allerdings nicht schon im Vorfeld bekannt sein.

Nach weiteren 30 Minuten finden wir uns zu einer Reflexionsrunde auf der Wiese vor ,Napulis Baum'[9] zusammen. Diese ist in zwei Teile geteilt: Einerseits interessiert uns, wie der Ort wahrgenommen wurde, welche Merkmale er hat, wie sich die Beteiligten verortet haben, und nehmen damit Bezug auf unsere ,Anleitung'. Außerdem möchten wir das Experiment als Experiment gemeinsam reflektieren. Die Diskussion zeichnen wir als Audioaufnahme auf, die Zitate in diesem Text stammen aus dem Transkript.

\section{Urban Citizen Walker: Reflexion}

Aus den ersten gehenden Erkundungen am Oranienplatz kristallisieren sich Themen heraus, die sowohl für die folgenden Beschreibungen und Analysen des Platzes als auch für Fragen nach der subjektiven Verortung im städtischen Raum grundlegend und bereichernd sind. In den vielstimmigen Verknüpfungen mit Erfahrungen von anderen Orten ebenso wie im Austausch des jeweiligen Wissens und der individuellen Ordnungs- und Erklärungsmuster zeigt sich die beeindruckende Komplexität urbaner Räume. Vor allem aber werden heterogene, auch widerstreitende Formen der Aneignung und Verortung im städtischen Raum greifbar. 
Im Folgenden zeigen wir einige Ausschnitte aus dem Transkript der Reflexionen zu Geschichte und Gestaltung des Ortes, seiner Bedeutung als öffentlichem Raum, als Symbol des Protestes und der alltäglichen Diversität. Darüber hinaus sind in der Reflexion Kommentare des In-Beziehung-Setzens mit eigenen Erfahrungen, der Selbstverortung und Fragen zur Stadtgesellschaft präsent.

\section{Erkundungen des O-Platzes}

Mitten in Kreuzberg 36 gelegen, ist der O-Platz immer wieder umkämpft gewesen, wurde wiederholt angeeignet und besetzt. Tarek erinnert sich an den Moment, als er das erste Mal nach Berlin kam und in einem Hostel fußläufig vom O-Platz wohnte:

„I thought all European cities would be like Prague, which is not a very diverse city, after three days here I re-configured my stereotypes, the cities are different." (Tarek)

Auch Erinnerungen an Demonstrationen, Straßenkämpfe und das zwischen 2012 und 2014 hier angesiedelte refugee camp sind vielen in unserer kleinen Gruppe in Erinnerung:

„I thought about the ghost of the refugee camp and also about the street riots. It is impossible for me to think about this place without thinking about these events, they were not one-time events but more cyclical..." (Agata)

„This is my first time ever, I just arrived in Berlin, I had a lot of feelings, but it is really interesting that so many events had happened here, it seems that it is a controversial space, I see a lot of contradictions that are significant, but I did not know about the movements here. Traces? I see the graffitis on the wall.“ (Randa)

Für Layla, die in Köpenick wohnt, ist die Diversität des O-Platzes eine neue, positive, fast beruhigende Erfahrung:

„I feel less as a Syrian here, always in Berlin... I feel like a stranger, I am a stranger, but it is very dominant, I feel it in every step. But here it is less." (Layla)

Die Diversität ist das, was den O-Platz ausmacht. Hier, so scheint es, ist es möglich, dass unterschiedliche Individualist*innen, Interessengruppen und Nutzungen zusammenfinden, ein Aushandeln von Raum fast lautlos stattfindet:

„Diversity gives meaning to everything: people, languages, colours, ages, different smells, sounds, everything, even the creatures, human beings, insects, animals - all my senses are occupied. [...] I can hear a lot of voices, the bells of the church, the bells of the bikes..." (Randa)

„... and about the languages, I heard so many languages, I do not know, English accent, Spanish, Armenian... Turkish... I hear rarely German.“ (Layla) 
„As I hear all this; this is a totally challenging space from the urban design, it is so vast, it has greenery, there is never some quiet area... there are streets, there are no hidden places..." (Martin)

Martin erzählt uns etwas zur städtebaulichen Entstehungsgeschichte:

„[Der Platz] [...] wurde als Teil eines größeren städtebaulichen Gefüges angelegt. Ursprünglich verlief hier ein Kanal, der die Spree mit dem Landwehrkanal verband. Geschlossen wurde er in den 1920er Jahren, nachdem schon im 19. Jahrhundert Moskitoplagen die Anwohner*innen mit Typhus infiziert hatten." (Martin)

Kathrin ergänzt mit Hinweisen zum sozio-ökonomischen Kontext:

„Anfang des 20. Jahrhunderts war der O-Platz sehr belebt, [...] ein Zentrum der rasant wachsenden Stadt, ein Ort der Produktion, des Konsums, mit Cafés, Billardsalons und Tanzhallen. [...] Während des Zweiten Weltkriegs wurden die Manufakturen zur Kriegsindustrie umgerüstet. Danach erfolgte eine Tabula rasa und viele der von den Bomben verschonten Häuser wurden abgerissen. [...] Mit der Mauer wurde der O-Platz zur Sackgasse und verlor seine Zentralität, war plötzlich ein Randgebiet. [...] Erst in den letzten zehn Jahren entwickelte er sich sukzessive (wieder) zu einem kreativen Hotspot. Heute ist die Gentrifizierung weit fortgeschritten. Vielleicht ist der Platz deshalb so faszinierend, weil hier trotzdem Platz für widerstände Praxis zu bleiben scheint.“ (Kathrin)

Wir sind uns einig, dass aus der Perspektive der Gestaltung der O-Platz schwierig zu nutzen ist. Die ihn durchkreuzende Oranienstraße teilt ihn in zwei Hälften. Weit auseinander installierte Bänke entlang der Mittelachse sind ein Relikt aus dem 19. Jahrhundert:

„Die Idee sollte sein, auf dem Platz zu promenieren, um sich dann zu zweit für einen Plausch niederzulassen.“ (Martin)

„Allerdings mit ausreichendem Abstand zur nächstgelegenen Sitzmöglichkeit, um die Privatsphäre der Gesprächspartner*innen zu schützen.“(Agata)[10]

Trotz dieser Herausforderungen und der Zäune um die Grünflächen, die ihre Nutzung erschweren, finden wir unterschiedlichste Formen der Aneignung. Außerdem, so findet Ayşe, lassen sich ,verschiedene Layer von Öffentlichkeit feststellen“ - sichtbare und weniger sichtbare, geplante und ungeplante:

„But to make a space a public space, you need to put public there. Well, I came here only for protest. [...] When I come here, I always feel lost, where to go, where is the address, where do I want to go? Only when I come to a protest which is starting here, I know I am at Oranienplatz. Then it is a public space, otherwise it is a transitionary space... it is not a space, a non-space [...] after the protest it leaves a whole emptiness behind [...]“ (Ayşe) 
„[...] but for me it is not emptiness, I do not see the non-belonging [...] I would never say it is a non-place, never [...] (Martin).

Randa und Layla, die keine Erinnerungen mit dem Platz verbinden, spüren doch dessen kontroverse Bedeutung und finden Spuren, die seine Geschichte(n) andeuten:

„Maybe it is a non-place for some people, and a meeting place for others. I do not want to look at people, I do not know about eye contact, I always keep my eyes down you never know...” (Layla)

Layla beobachtet, wie eine (vermutlich) Roma-Familie auf dem südlichsten Zipfel des O-Platzes eine Grünfläche okkupiert und Sperrmüll-Sessel aufgestellt hat, durch die ein Wohnzimmer oder ein Aufenthaltsraum angedeutet wird. Eine weitere Ausführung eines knallrosa Sessels in Muschelform verschwindet zusammen mit einem jungen (wahrscheinlich) Studenten im Bus, der dort hält, wo einst besagter Kanal verlief. Die Menschen, die aus den Bussen strömen, passieren den O-Platz auf dem Weg nach Hause. Teilweise beobachten wir, wie auf den Bänken Platz genommen wird, um eine Verschnaufpause einzulegen. Auch zufällige Begegnungen finden statt und Leute nutzen die Sitzgelegenheiten, wie geplant, zum Plauschen.

In der nordwestlichen Ecke des O-Platzes hat sich eine Gruppe wohnungsloser Menschen versammelt. Sie treffen sich unter einer Baumgruppe und sind dadurch weniger sichtbar, wirken eher ,in die Ecke gedrängt‘, jedoch, anscheinend, aus eigener Wahl. Zwischendurch werden die Bänke von Touristen*innen vereinnahmt, die hier ihren Stadtplan studieren und ihren weiteren Weg durch die Stadt planen.

Layla stellt fest, dass die Zweiteilung des Platzes ihr gefühltes Sicherheitsempfinden am Ort spiegelt:

„I feel there are two parts, here I do not feel safe, but on the other side, I can sit on the bench. As the pigeon, here is no pigeon, but over there, there are birds... here is nothing." (Layla)

Hişar entdeckt ein paar gebrauchte Fahrräder, die am nördlichsten Ende des O-Platzes, gegenüber vom Café Kuchenkaiser, an einen Zaun gekettet sind und mittels einer auf einem Stück Pappe notierten Telefonnummer zum Verkauf angeboten werden. Vielleicht findet an dieser Stelle der informelle Handel statt?

Die ,Geister der Vergangenheit', etwa Proteste und Okkupationen, und ihre politischen Dimensionen, sind auch heute noch spürbar. Und sie werden mittels hinterlassener Spuren in Erinnerung gerufen. Katharina liest einen per Hand geschriebenen Schriftzug auf einer Bank: „Lübcke - Die AFD hat mit geschossen."

Somit werden wir mit der politischen Realität in unserem Land konfrontiert und die (andauernden) Kämpfe von geflüchteten Menschen um Teilhabe in den Blick gerückt.

Die Vielzahl an Eindrücken und Emotionen, Entdeckungen und Erfahrungen werfen die Frage auf, was wir durch das gemeinsame Gehen über den konkreten Ort, den O-Platz, und über Stadt im Allgemeinen, aber auch über uns und unsere Assoziationen und Erinnerungen erfahren. Welche 
unterschiedlichen Perspektiven eröffnen sich? Daran anschließen lässt sich die Frage, wie das (gemeinsame) Gehen als strukturierte Methode der kritischen Stadtforschung weiterentwickelt werden kann, um die Komplexität von Stadt vor dem Hintergrund unterschiedlicher Erfahrungen zu analysieren.

\section{Reflexionen zum Experiment}

„Maybe we are trying to see too much...“(Ayşe)

Der O-Platz erschien uns prädestiniert, um über Aneignungsprozesse und Urban-Citizenship-Praktiken ins Gespräch zu kommen. Es zeigte sich, dass es auch an einem Ort, der nicht allen unbekannt und folglich mit Assoziationen und Erinnerungen bereits besetzt ist, noch viel zu entdecken und Neues, Unerwartetes zu sehen, hören, fühlen und zu ertasten gibt. Die Bandbreite an Themen, die angesprochen wurden, verdeutlicht dies. Das Gehen an diesem Ort sensibilisierte uns in der Wahrnehmung und für die verschiedenen Bedeutungen von Stadt. Auch der ,Überraschungseffekt‘, dass die Teilnehmende weder über den Ort noch das Vorgehen im Vorhinein informiert wurden, wurde positiv bewertet; Anregungen gab es zur Interdisziplinarität der Gruppe sowie zu einer möglichen (historischen) Kontextualisierung:

"I liked the not knowing part, but the group could be even more diverse, musicians, architects... at the end maybe a brief historical input.“ (Layla)

Spannend ist, dass das Gehen allein für einige der Urban Citizen Walker besondere Emotionen anrührte und Erinnerungen hervorbrachte, zum Teil angestoßen durch einfache Gegebenheiten wie Materialien und Oberflächen, die Assoziationen hervorriefen:

„I had a lot of emotions and visions, but I am an alien, I see things for the first time, I just see the things as they are. I started to visualise my country [...] this space which is not paved, the sandy part reminded me of a space I went with my family on weekends to picnic..." (Randa)

Auch der Kontakt zu sich selbst wurde durch das Gehen ermöglicht:

„Everyone seems occupied with his own self, I was happy to have time to reflect on that, I like this exercise because it makes you calm down, be closer to yourself..." (Randa)

Beim Gehen zu zweit wurden Verknüpfungen zu anderen Orten und Erinnerungen hergestellt und dadurch neue Sichtweisen für den*die Mitspazierende* $\mathrm{n}$ auf den O-Platz eröffnet:

„[...] especially interesting to hear the different perspectives... Walking with two persons, Kate... looking with the Athens lens on the place... connecting it to your experiences and knowledges of other places." (Martin)

Jetzt bleibt die Frage, wie sich dieses kollaborative Experiment als Methode in die gegenwärtige Stadtforschung einspeisen lässt. 


\section{Die Methode des kollaborativen Gehens und Teilhabe an Stadtgesellschaft}

Gehen wir noch einmal zurück an den Anfang und zu unseren konzeptionellen methodologischen Überlegungen: Wodurch zeichnet sich das gemeinsame Gehen aus? Und wie lässt es sich als Methode der kritischen Stadtforschung weiterentwickeln, um die Komplexität von Stadt vor dem Hintergrund unserer unterschiedlichen individuellen Erfahrungen zu analysieren und damit neue städtische Räume zu öffnen? Aus den theoretischen, konzeptionellen Überlegungen und den Erfahrungen des Experiments ergeben sich zunächst drei Ebenen der Analyse, die wir im Folgenden darstellen und reflektieren.

\section{In Situ - Being (t)here}

Zunächst ist da die Bedeutung der konkreten Erfahrung von Raum und Zeit. Gehen findet in der Gegenwart statt. Das Gehen ist an einen konkreten Ort gebunden. Die Urban Citizen Walker beobachten den Ort und setzen sich, durch ihre Erfahrungen an diesem oder an anderen Orten, zu ihm in Beziehung. Sie agieren gehend, eine aktive oder auch performative Handlung, durch die der städtische Raum - ganz im Sinne einer Rhetorik des Gehens wie oben mit de Certeau (1980) und Augoyards (2007) beschrieben - produziert wird (vgl. Rolshoven 2017). Diese Handlungen und Erfahrungen schreiben sich wiederum in den Raum ein, sind Teil des gelebten Raumes der Alltagspraxis (vgl. Lefebvre 1991). So entsteht über das Gehen im ,Hier und Jetzt' hinaus eine Verknüpfung mit eigenen Erfahrungen sowie mit Vorstellungen von städtischem Raum und dessen Funktionen. Durch die Anwesenheit vor Ort und die eigenständige sowie kollektive Reflexion lassen sich komplexe Schichten eines konkreten urbanen Raumes entblättern. Es findet ein Austausch statt, wodurch die Einzelnen zu einem kollektiven Wissen beitragen.

\section{Körperlichkeit und Subjektivität}

Das Gehen basiert auf einer subjektiven, physischen Erfahrung des Körpers im Raum. Erinnerungen tauchen auf, Einschreibungen werden erfahrbar. Wie in den Experimenten der situationistischen Psychogeographie (u. a. Debord 1990) ist das gehende Individuum durch seinen*ihren Körper mit dem ihn* sie umgebenden Raum konfrontiert. Dabei wirkt der Raum konkret auf den Körper und vice versa. Es handelt sich um eine Zeit- und Raumerfahrung durch die Bewegung des Körpers. Die subjektiven Erfahrungen werden als qualitative Daten der Stadtwahrnehmung interpretiert, die als empirische Wahrnehmungstechnik Facetten des Raumes versammelt (Rolshoven 2017). Im kollaborativen Gehen in der Gruppe wird der Körper zu einem gemeinsamen Wissenskorpus.

\section{Teilhabe an Stadt}

Schließlich ist da eine politische Dimension des Gehens: Die Forschenden (inter)agieren im und mit dem Ort. So wird die Anwesenheit der (forschenden) Gruppe zu einem performativen Moment der Situation (vgl. 
Hildebrandt 2014). Das Gehen als Methode der Stadtforschung kann im Sinne von John Law und John Urry (2004: 391) als eine soziale, räumliche und politische Praxis eingesetzt werden, die die soziale Wirklichkeit nicht nur beschreibt, sondern sie auch aufführt, das heißt enacted. Die Gehenden als Forschende positionieren sich im und zum Raum und nehmen damit selbst an der Produktion von Stadt teil. Mit McFarlane ist das Gehen nicht nur ein Bewusstwerden oder Lernen des urbanen Raumes, sondern muss als eine temporär verortete Praxis verstanden werden (McFarlane 2011: 50). Sich gehend im Raum zu bewegen, bedeutet also immer auch eine Art der Raumaneignung (Lisiak et al. 2019). Dementsprechend öffnet die Methode des kollaborativen, engagierten Gehens im Sinne einer widerständigen Alltagspraxis (Winkler 2017) Möglichkeiten, Räume (neu) zu besetzen und sich im (gesellschaftlichen) Raum zu positionieren. Gehen ist damit ein Instrument der aktiven und selbstermächtigten lokalen Verortung, eine (kollektive und performative) Aneignung städtischer öffentlicher Räume und damit der Teilhabe an Stadt und ein „act of citizenship“ (vgl. Irazabal 2008; Isin 2017).[11]

\section{Keep on walking}

Das hier vorgestellte Experiment sowie weitere kollaborative Gehversuche und deren Reflexion eröffnen vielfältige Dialoge zwischen Praxis, Forschung und Intervention und somit neue Perspektiven auf Stadt. Ein Ziel ist es, mit den Urban Citizen Walks einen (performativen) Raum der Erfahrung und des Austauschs zu schaffen, in dem über Teilhabe an Stadt verhandelt und somit selbstermächtigtem politischem Handeln Platz gegeben werden kann.

Mittels solcher kollektiver walks projizieren wir unsere Wünsche und entwickeln gemeinsame Visionen für die Stadt. Wie hier dargestellt, war das gemeinsame Gehen am O-Platz für uns ein Experiment mit dem Ziel, zukünftig regelmäßige Urban Citizen Walks zu etablieren. Wichtig ist uns, dass auch Andere sich stimuliert fühlen, die Idee aufzugreifen und Urban Citizen Walks zu initiieren. Eine Reihe von Ideen und Anregungen für zukünftige walks haben sich bereits in der Feedback-Runde ergeben:

„Walk to disturb, trigger something else... make interventions... small things,

Maybe invent a street fight...,

A topic like public space and politics...,

Walk with different kind of ,glasses/lenses'... as a metaphor but as well as real objects,

Try different movements,

Repeat walks on one spot, different perspectives different times... at night,

Rooftops, thinking about different levels,

Do the same at other places, with the same topic... publicness...

Peripheries, mass housing projects..."

(Auszüge aus der Abschlussdiskussion mit der Gruppe der Urban Citizen Walker) 


\section{Endnoten}

[1] Zum Begriff des urban practioners als transdisziplinäre aktive Rolle in der gegenwärtigen Stadt vgl. Holub (2015).

[2] „How do we live together?“ wurde von den Architekt*innen Ingrid Sabatier und Stephan Schwarz und der urban practitioner Katharina Rohde initiiert und für das DAZ kuratiert. Gefördert wurden die Stadtspaziergänge durch den Berliner Projektfond Kulturelle Bildung im Kontext des Projektes Berlin Mondiale.

[3] http://refugeevoicestours.org/ (letzter Zugriff am 18.11.2020)

[4] Aus der Vielzahl von wissenschaftlichen Texten zum Gehen in der Stadt siehe u. a. Burckhardt (2011), Kusenbach (2003), Rolshoven (2017), Thibaud (2017), Winkler (2017).

[5] Zu künstlerischen Praktiken siehe beispielsweise Arbeiten von Francis Alys, der mit seinem Gehen die Stadt erkundet und in sie interveniert: „Paradox of Praxis - Sometimes Making Something Leads to Nothing“, Mexico City 1997 (https://francisalys.com/sometimesmaking-something-leads-to-nothing/, letzter Zugriff am 18.11.2020) oder auch das aktuelle Ausstellungsprojekt „Des marches, démarches“ im Frac Marseille, kuratiert von Guillaume Monsaingeon (https://www.fracpaca.org/des-marches-demarches, letzter Zugriff am 18.11.2020).

[6] In der deutschsprachigen Stadtforschung nur wenig rezipiert, gibt es in französischsprachigen Stadtforschung über Michel de Certeau hinaus eine ganze Reihe von Forscher*innen, die das Gehen als Methode der Exploration und der Wahrnehmung, als Element der teilnehmenden Beobachtung als ein zentrales Forschungsinstrument einsetzen, vgl. hierzu u. a. Winkler (2016) sowie die Arbeiten von Augoyard (2007 [1979]).

[7] „[...] kurzes Innehalten und sich Aufhalten, Zäsuren im Parcours gewähren Ausblicke, Auswege aus den vorgegebenen Sichtweisen, Brüche unserer Darstellungen.“ (Rolshoven 2017:101)

[8] $\mathrm{Zu}$ der Protestbewegung von geflüchteten Menschen und deren Aneignung des O-Platzes vgl. u. a. Fontanari (2016) und Lanz (2015).

[9] Als Protest gegen die Räumung des selbstorganisierten Camps auf dem O-Platz im April 2014 verbrachte Napuli Paul Langa, eine der Aktivist*innen der Bewegung, fünf Tage auf einen Baum am O-Platz und protestierte gegen die Räumung (vgl. Fontanari 2016).

[10] Im Frühjahr 2020 und mit den aktuellen Erfahrungen des ,social/physical distancing als Antwort auf Covid-19 scheint dieser ,Sicherheitsabstand' fast surreal, erzeugt jedoch bei längerem Verweilen etwas Spannendes: So ermöglicht das Sitzen auf den Bänken ein distanziertes Beobachten und gleichzeitige Teilhabe am unmittelbaren Leben auf dem Platz.

[11] In Anlehnung an Engin Isins Verständnis von „acts of citizenship as quotidian enactments, which might lack the visibility of certain performative acts but nevertheless can be consequential“ (Isin 2017: 509).

\section{Autor_innen}

Katharina Rohde arbeitet international als Urban Practitioner und forscht aktuell im Rahmen ihrer Dissertation zu Migrant Agency in Berlin und Johannesburg. Ihre Themenschwerpunkte sind Migration, Agency und Urbane Praxis.

me@katharina-rohde.com

Kathrin Wildner ist Stadtethnologin. Sie forscht, lehrt und publiziert zu qualitativen und künstlerischen Methoden der Stadtforschung.

kwildner@zeromail.org 


\section{Literatur}

Arendt, Hannah (1992) The Human Condition. Chicago: University of Chicago Press.

Augoyard, Jean-Francois (2007 [1979]): Step by Step. Everyday Walks in a French Urban Housing Project. Minneapolis: University of Minnesota.

Augoyard, Jean-Francois (2017): Die Rhetorik des Wohnens: Gehfiguren. In: Justin Winkler (Hg.), Gehen in der Stadt. Ein Lesebuch zur Poetik und Rhetorik des städtischen Gehens. Weimar: Jonas Verlag, 26-63.

Benjamin, Walter (1998): Das Passagenwerk. Gesammelte Schriften Bd. V. Frankfurt am Main: Suhrkamp.

Benjamin, Walter (2015): Stadt des Flaneurs. Berlin: be.bra.

Brown, Evrick / Shortell, Timothy (2016): Walking in Cities. Quotidian Mobility as Urban Theory, Method, and Practice. Philadelphia: Temple University Press.

Cadogan, Garnette (2016): Walking While Black. https://lithub.com/walking-while-black/ (letzter Zugriff am 18.11.2020).

de Certeau, Michel (1988): Kunst des Handelns. Berlin: Merve.

Cole, Teju (2012): Open City. Berlin: Suhrkamp.

Debord, Guy (1990): Theorie des Umherschweifens. In: Der große Schlaf und seine Kunden. Situationistische Texte zur Kunst. Hamburg: Nautilus, 33-40.

Dündar, Özlem Özgül / Göring, Mia / Othmann, Ronya / Sauer, Lea (Hg.) (2019): flexen. Flaneusen* schreiben Städte. Berlin: Verbrecher.

Fischer, Ralph (2011): Walking Artist - Über die Entdeckung des Gehens in den performativen Künsten. Bielefeld: transcript.

Fontanari, Elena (2016): Looking for Neverland. The Experience of the Group „Lampedusa in Berlin" and the Refugee Protest of Oranienplatz. In: Gökce Yurdakul / Regina Römhild / Anja Schwanhäußer / Birgit zur Nieden (Hg.), Witnessing the Transition. Moments in the Long Summer of Migration. Berlin: Assoziation A, 15-35.

Foroutan, Naika (2019): Die postmigrantische Gesellschaft. Ein Versprechen der pluralen Demokratie. Bielefeld: transcript.

Garcia Canclini, Nestor (1995): Consumidores y ciudadanos. Conflictos multiculturales de la globalización. Mexiko Stadt: Editorial Grijalbo.

Grube, Nils / Thiele, Katja (2020): Kritische Stadtexkursionen. Annäherung an eine reflexive Exkursionspraxis. In: sub \urban. zeitschrift für kritische stadtforschung 8/3, 215-230.

Hessel, Franz (2011): Spazieren in Berlin. Berlin: Berlin Verlag Taschenbuch.

Hildebrandt, Paula Marie (2014): Staubaufwirbeln. Oder die Kunst der Partizipation. Weimar: Bauhaus-Universität, Dissertation.

Holub, Barbara (2015): Planning Unplanned. Towards a New Positioning of Art in the Context of Urban Development. Wien: Verlag für moderne Kunst.

Ingold, Tim / Vergunst, Jo Lee (Hg.) (2008): Ways of Walking. Ethnography and Practice on Foot. London: Taylor \& Francis.

Irazabal, Clara (2008): Citizenship, Democracy, and Public Space in Latin America. In: Clara Irazabal (Hg.), Ordinary Places, Extraordinary Events. Citizenship, Democracy, and Public Space in Latin America. New York: Routledge, 11-35.

Isin, Engin (2017): Performative Citizenship. In: Ayelet Shachar / Rainer Bauböck / Irene Bloemraad / Maarten Vink (Hg.), Oxford Handbook of Citizenship. Oxford: Oxford University Press, 500-523.

Krakauer, Siegfried (1964): Straßen in Berlin und anderswo. Frankfurt am Main: Suhrkamp.

Kusenbach, Margarethe (2003): Street phenomenology. The go-along as ethnographic research tool. In: Ethnography 4/3, 455-485.

Lanz, Stephan (2015): Refugees und die Stadt. In: Arno Brandelhuber / Florian Herzwech / Thomas Mayfried (Hg.), The Dialogic City. Berlin wird Berlin. Berlin: Walter König, 487-495.

Law, John / Urry, John (2004): Enacting the Social. Economy and Society 33/3, 390-410.

Lefebvre, Henri (1991): The Production of Space. Hoboken: Wiley-Blackwell.

Lisiak, Agata / Cox, Reece / Tienes, Flavia M. / Zbinovsky Braddel, Sophia (2019): A city coming into being. Walking in Berlin with Franz Hessel and Marshall Berman. In: CITY 22/5, $1-17$. 
McFarlane, Colin (2011): Learning the City. Knowledge and Translocal Assemblage. Oxford: Wiley-Blackwell.

Nguyen, Thien Vinh (2016): Learning to Walk. On curating a walking-methodologies programme. In: Ben Campkin / Ger Duijzings (Hg.), Engaged Urbanism. Cities and Methodologies. London/New York: IB Tauris, 187-198.

Pétonnet, Colette (2017): Variationen über das Rauschen einer Dauerbewegung, In: Justin Winkler (Hg.), Gehen in der Stadt. Ein Lesebuch zur Poetik und Rhetorik des städtischen Gehens. Weimar: Jonas Verlag, 63-75.

Rohde, Katharina / d'Auria, Viviana (2018): Building Cosmopolitan Citizenship Through the Mundane. Insights from Walking Conversations in the Public Spaces of Berlin. In: European Journal of Creative Practices in Cities and Landscapes 1/1, 33-52.

Rolshoven, Johanna (2017): Gehen in der Stadt. In: Justin Winkler (Hg.), Gehen in der Stadt. Ein Lesebuch zur Poetik und Rhetorik des städtischen Gehens. Weimar: Jonas Verlag, 95-112.

Sadler, Simon (1999): The Situationist City. Cambridge, Mass.: MIT Press.

Solnit, Rebecca (2000): Wanderlust. Eine Geschichte des Gehens. Berlin: Matthes \& Seitz.

Streule, Monika (2018): Ethnografie urbaner Territorien. Metropolitane Urbanisierungsprozesse von Mexiko-Stadt. Münster: Westfälisches Dampfboot.

Thibaud, Jean-Paul (2017): Über die Daseinsweise des Gehens in der Stadt. In: Justin Winkler (Hg.), Gehen in der Stadt. Ein Lesebuch zur Poetik und Rhetorik des städtischen Gehens. Weimar: Jonas Verlag, 112-129.

Winkler, Justin (2017): Zur Einführung. Gehen als widerständige Alltagspraxis. In: Justin Winkler (Hg.), Gehen in der Stadt. Ein Lesebuch zur Poetik und Rhetorik des städtischen Gehens. Weimar: Jonas Verlag, 11-17. 